\title{
Prematuros moderados y tardíos, un grupo de riesgo de menor desarrollo cognitivo en los primeros años de vida
}

\author{
LUISA SCHONHAUT B. ${ }^{1}$, MARCELA PÉREZ R. ${ }^{1}$, MARIANNE SCHONSTEDT G. ${ }^{2}$, \\ IVÁN ARMIJO R. ${ }^{3}$, IRIS DELGADO B. ${ }^{4}$, MIGUEL CORDERO V. ${ }^{5}$, JORGE ÁLVAREZ L. \\ 1. Pediatra, Departamento de Pediatría, Facultad de Medicina Clínica Alemana-Universidad del Desarrollo. \\ 2. Terapeuta Ocupacional, Departamento de Pediatría, Facultad de Medicina Clínica Alemana - Universidad del Desarrollo. \\ 3. Psicólogo, Escuela Psicología Universidad del Desarrollo. \\ 4. Bioestadístico, Facultad de Medicina Clínica Alemana-Universidad del Desarrollo. \\ 5. Psicólogo, Ministerio de Salud-Chile.
}

\begin{abstract}
Moderately and late preterm newborns, a risk group for low cognitive development in the first years of life

Introduction: Recent studies show that moderately and late preterm infants (MLI) (Gestational age (GA) 32 to $36^{6}$ ), are in high risk for delayed psychomotor development (PMD) and learning disabilities. Objective: To compare PMD between MLI and full term infants (FTI) and to analyze associated perinatal risk factors. Methodology: 131 MLI and 119 FTI were recruited between May 2008 and April 2011 in a private health center in Santiago, Chile. Both groups were compared according to age, gender and socio-economic level. At the ages of 8, 18 and 30 months the children were evaluated by means of the Bayley III Scale for Infantile Development $3^{\text {rd }}$ edition (BAYLEY-III). PMD was compared in both groups and multiple lineal regression analysis was performed. Results: MLI showed a significantly lower development coefficient, but it matched the FTI when corrected by GA, $(99 \pm 10.4$ versus $100.8 \pm 9.9)$. Even with corrected GA, the cognitive performance was lower (98 \pm 10.4 vs $103.1 \pm 11.4 \mathrm{p}<0.05)$. Multiple lineal regressions showed that GA and gender were associated with lower cognitive development. Conclusion: Cognitive performance of MLI was below that of FTI. This increases the need to have especial standards of care and stimulation for these children.

(Key words: Moderately preterm, late preterm, psychomotor development, cognitive).

Rev Chil Pediatr 2012; 83 (4): 358-365
\end{abstract}

\section{RESUMEN}

Introducción: Estudios recientes refieren que los prematuros moderados y tardíos (PMT) (Edad Gestacional (EG) 32 a $36^{6}$ ), presentan un elevado riesgo de retraso del desarrollo psicomotor (DSM) y dificultades de aprendizaje. Objetivo: Comparar el DSM entre niños nacidos PMT y recién nacidos de término (RNT) y ana-

Proyecto con financiamiento SOCHIPE 208013, FONIS SA7120043 y Clínica Alemana de Santiago.

Recibido el 28 de noviembre de 2011, devuelto para corregir el 05 de marzo de 2012, segunda versión el 20 de marzo de 2012 , aceptado para publicación el 9 de abril de 2012.

Correspondencia a:

Luisa Schonhaut Berman

E-mail: Ischonhaut@alemana.cl 
lizar los factores de riesgo perinatal asociados. Metodología: En un centro de salud de Santiago de Chile, entre mayo 2008 y abril 2011, se reclutaron 131 PMT y 119 RNT, pareados por edad, género y nivel socioeconómico. A los 8, 18 o 30 meses se les aplicó la Escala de Bayley III de Desarrollo Infantil-3ª edición. Se comparó el DSM entre los dos grupos y se implementó una regresión lineal múltiple. Resultados: Los PMT presentaron un coeficiente de desarrollo significativamente inferior al no corregir EG, equiparándose con los RNT al corregirla (99 $\pm 10,4$ vs 100,8 \pm 9,9). Aún con EG corregida, el desempeño cognitivo fue inferior ( $98 \pm 10$,4 vs $103,1 \pm 11,4$ p < 0,05). En la regresión lineal múltiple el género y la EG se asociaron con menor desarrollo cognitivo. Conclusión: El desempeño cognitivo de los PMT fue inferior a los RNT. Se plantea la importancia de implementar un estándar de cuidado y estimulación para este grupo de niños.

(Palabras clave: Prematuros moderados, prematuros tardíos, desarrollo psicomotor, cognitivo).

Rev Chil Pediatr 2012; 83 (4): 358-365

\section{Introducción}

Los prematuros moderados y tardíos (PMT), es decir aquellos niños que nacen entre las 32 y las $36^{6}$ semanas de gestación, representan aproximadamente el 10\% del total de partos en USA durante la última década ${ }^{1}$ y cerca del $6 \%$ de los partos en nuestro país².

Dado que, a lo largo de la historia, la tasa de mortalidad de los prematuros ha ido en franco descenso, los de mayor peso de nacimiento han sido considerados libres de riesgo, continuando con los cuidados pediátricos habituales. En Chile y otros países los programas de seguimiento están dirigidos a los prematuros extremos, es decir, con EG $<32$ semanas o peso de nacimiento inferior a $1500 \mathrm{gr}^{3}$.

La renovada atención por los prematuros tardíos data del año 2005, en que se generó un consenso mundial respecto a considerarlos como un nuevo grupo de riesgo biológico dado que, al compararlos con los recién nacidos de término (RNT), presentan mayores tasas de morbilidad tanto a corto como a largo plazo ${ }^{4-6}$. En el período neonatal presentan mayor probabilidad de hospitalización debido a problemas biomédicos, tales como hiperbilirrubinemia, hipoglicemia, dificultad respiratoria, problemas de alimentación y apneas, entre otros ${ }^{7,8}$. Mientras que a largo plazo, la mayoría de los estudios señalan que los PMT presentan prevalencias de déficit del desarrollo psicomotor (DSM), dificultades de aprendizaje y problemas de comportamiento superiores a los niños nacidos de término ${ }^{9-13}$.

No obstante los resultados globales señalan el riesgo, quedan numerosas controversias por resolver; por ejemplo, respecto a la necesidad de corregir la EG al momento de la evaluación del DSM: el estudio de Romeo indica que el rendimiento de los PMT se equipararía a los RNT al corregir la EG a los 12 y 18 meses $^{14}$, corrección que no todos consideran al momento de evaluarlos. También cabe cuestionarse si los posibles problemas del desarrollo y aprendizaje son atribuibles exclusivamente a la prematuridad o son el producto de la sumatoria de factores de riesgo asociados a ésta, como el menor peso de nacimiento o la morbilidad neonatal ${ }^{15-17}$. Gurka reporta que los prematuros tardíos de familias de mayor nivel socio económico y que no requirieron hospitalización en el período neonatal, tienen un desempeño similar a los niños nacidos de término ${ }^{18}$, por otro lado, Kalia demuestra que los PMT con comorblidad y hospitalización neonatal, tienen similar probabilidad de requerir servicios de apoyo al desarrollo que los prematuros extremos ${ }^{19}$.

\section{Objetivo}

Comparar el desempeño, en las distintas áreas del DSM, de niños nacidos PMT con RNT y analizar los factores de riesgo perinatal asociados.

\section{Metodología}

\section{Población participante}

Estudio analítico prospectivo, de lactantes y preescolares de 8, 18 ó 30 meses de EG corregida, nacidos en un centro privado de la Región Metropolitana de Santiago de Chile, entre los años 2007 y 2009. Cada niño fue evaluado en una sola oportunidad en el estudio. 
En base a lo documentado, supusimos una diferencia de $12 \%$ en el promedio de coeficiente de desarrollo entre los PMT y los RNT y una desviación estándar esperada de 30\% y $25 \%$ respectivamente para cada grupo. Considerando un intervalo de confianza de 95\% y una potencia de $90 \%$, se estimó un tamaño muestral de 112 niños por grupo.

El grupo de estudio estuvo conformado por lactantes y preescolares nacidos entre las 32 y $33^{6}$ semanas de EG, que son los prematuros moderados y entre las 34 y $36^{6}$ semanas de EG o prematuros tardíos. Para nuestro análisis consideramos todo el grupo como PMT. La determinación de la EG se realizó en base a la fecha de última regla y/o una ecografía precoz ( $<12$ semanas).

El grupo control fue conformado por niños nacidos de término, pareados por edad, género y nivel socioeconómico.

Se excluyeron los lactantes y preescolares con diagnóstico conocido de alteración en el DSM, enfermedad genética, metabólica o neurológica conocida.

\section{Enrolamiento}

Los padres de los pacientes fueron contactados telefónicamente, aceptando participar a través de un consentimiento informado. Aproximadamente el $90 \%$ de los padres y niños contactados participó en la evaluación del DSM.

La información biodemográfica y médica se obtuvo de un formulario completado por los padres o cuidadores principales. En caso de los niños que estuvieron hospitalizados en el período neonatal, se revisaron las fichas clínicas correspondientes.

Los niños fueron evaluados mediante la Escala de Bayley III de Desarrollo Infantil $3^{\text {a }}$ edición, 2005 (Bayley-III) ${ }^{20}$, aplicada por una terapeuta ocupacional capacitada, calculando los puntajes del niño con y sin corregir EG. Para la corrección de la EG se restaron las semanas faltantes al nacimiento para llegar a las 40 semanas.

Los resultados de la evaluación fueron enviados a los pediatras tratantes, y en los casos en que se encontró dificultades del DSM, se sugirió una evaluación o intervención adicional, individualizada para cada niño.

\section{Descripción del instrumento}

Bayley-III: Escala que evalúa el desarrollo en niños de 1 a 42 meses. Consta de tres subescalas diferenciadas, que miden separadamente tres áreas del desarrollo: cognitiva, comunicación (comprensiva y expresiva) y motora (gruesa y fina). Los puntajes totales de cada subescala fueron llevados a puntajes estándar, donde la media es 100 puntos y 1 DS es 15 puntos.

\section{Comité de ética}

El estudio contó con la aprobación del Comité de Ética de la Investigación de Facultad de Medicina Clínica Alemana-Universidad del Desarrollo.

\section{Análisis estadístico}

Se realizó un análisis descriptivo de los puntajes obtenidos, estratificados por edad. Para las variables categóricas se aplicó test exacto de Fisher. La comparación de los promedios de las variables continuas, se analizó mediante ANOVA y su asociación se realizó a través del test de correlación de Pearson.

Finalmente se implementó un modelo de regresión lineal, cuya variable dependiente fue el desempeño en las distintas áreas del desarrollo, y las variables independientes fueron todas aquellas que resultaron significativas en el análisis bivariado $(\mathrm{p}<0,05)$.

El análisis estadístico se realizó con el software SPSS versión 18.0 y el cálculo de la muestra con EPIDAT 3.1.

\section{Resultados}

Se reclutaron 119 RNT y 131 PMT. Al momento de la evaluación ambos grupos fueron equivalentes en edad post-natal (corregida para PMT), género, nivel socioeconómico, años de educación y edad materna. Hubo diferencias significativas en las variables perinatales relacionadas con la prematuridad, como la hospitalización neonatal, el peso de nacimiento, peso para el EG y gestación múltiple (tabla 1).

Los PMT presentaron un coeficiente de desarrollo significativamente inferior al no corregir EG $(92,9 \pm 10,4$ vs 100,8 \pm 9,9, $\mathrm{p}<0,01)$, equiparándose con los RNT al corregirla (99 \pm 
Tabla 1. Características biomédicas y demográficas de los niños que nacieron de término y los nacidos entre las 32 y 36 semanas de edad gestacional

\begin{tabular}{|c|c|c|c|}
\hline & $\begin{array}{l}\text { Recién nacidos de } \\
\text { término }\end{array}$ & $\begin{array}{c}\text { Prematuros de } 32 \text { a } 36 \text { semanas } \\
\text { de edad gestacional }\end{array}$ & $\mathbf{p}$ \\
\hline $\mathrm{n}$ & 119 & 131 & \\
\hline $\begin{array}{l}\text { Edad evaluación } \\
\qquad 8(n) \\
18(n) \\
30(n)\end{array}$ & $\begin{array}{l}43 \\
39 \\
37\end{array}$ & $\begin{array}{l}47 \\
43 \\
41\end{array}$ & NS \\
\hline Género masculino, n (\%) & $57(43,1 \%)$ & $74(56,9 \%)$ & 0,086 \\
\hline EG (Prom. \pm DS) & $38,9 \pm 0,7$ & $33,9 \pm 1,4$ & $P<0,0001$ \\
\hline PN (Prom. \pm DS) & $3259 \pm 374,5$ & $2125,23 \pm 461$ & $P<0,0001$ \\
\hline PEG n (\%) & $7(8 \%)$ & $56(42,7 \%)$ & $P<0,0001$ \\
\hline Gemelar n (\%) & $2(1,7 \%)$ & $65(49,6 \%)$ & $P<0,0001$ \\
\hline Hospitalización n (\%) & $5(4,2 \%)$ & $111(84,7 \%)$ & $P<0,0001$ \\
\hline Edad Materna (Prom. \pm DS) & $33,8 \pm 3,8$ & $34,5 \pm 3,6$ & NS \\
\hline Años Educación Materna (Prom. \pm DS) & $17,8 \pm 2,7$ & $17,3 \pm 2,3$ & NS \\
\hline Ingreso decil 8-9 (\%) & $96 \%$ & $95 \%$ & NS \\
\hline
\end{tabular}

Prom.$=$ Media de puntaje, DS $=$ desviación estándar. EG $=$ edad gestacional, PN $=$ Peso nacimiento, PEG $=$ Pequeño para la edad gestacional.

Tabla 2. Comparación de puntaje promedio de la Escala de Bayley-III de Desarrollo Infantil de los prematuros moderados y tardíos con los recién nacidos de término según área

\begin{tabular}{|lccr|}
\hline Área del desarrollo & $\mathbf{3 2 - 3 6}$ EG-NC & $\mathbf{3 2 - 3 6}$ EGC & RNT \\
\hline $\mathrm{n}$ & 131 & 131 & 119 \\
\hline Cognitivo (Prom. \pm DS) & $92,1 \pm 10,3^{*}$ & $98 \pm 10,4^{*}$ & $103,1 \pm 11,4$ \\
Lenguaje (Prom. \pm DS) & $95,6 \pm 13,4^{*}$ & $101,5 \pm 13,8$ & $102,8 \pm 12,6$ \\
Motor (Prom. \pm DS) & $91,1 \pm 13,6^{*}$ & $99,5 \pm 13,7$ & $96,9 \pm 13,8$ \\
\hline Promedio (Prom. \pm DS) & $92,9 \pm 10,3^{*}$ & $99 \pm 10,4$ & $100,8 \pm 9,9$ \\
\hline
\end{tabular}

${ }^{*} \mathrm{p}<0,01$. Prom. $=$ promedio; DS=Desviación estándar. EG-NC = Edad gestacional no corregida, EGC = Edad gestacional corregida, RNT= Recién nacidos de término.

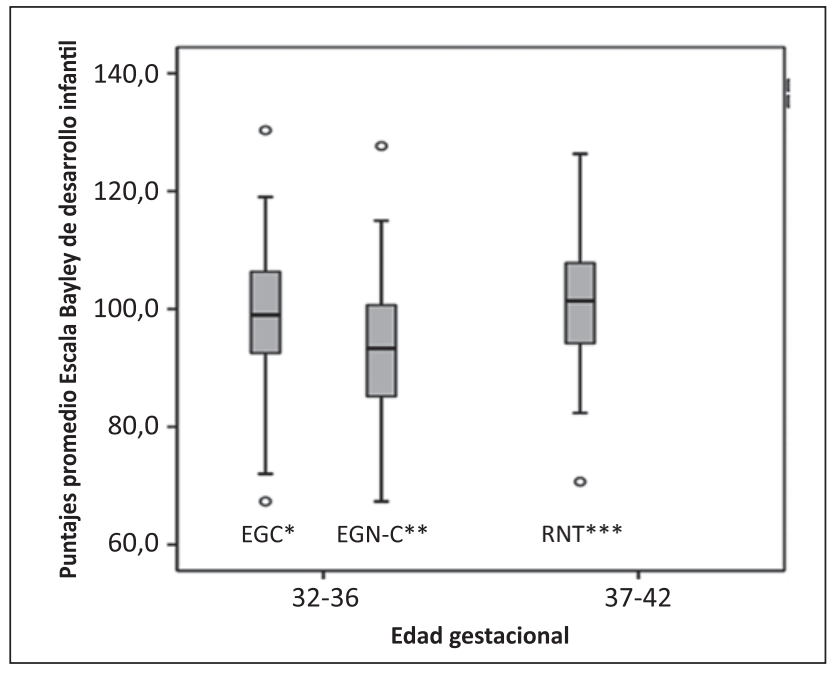

Figura 1. Análisis comparativo del puntaje de la Escala de Bayley-III de Desarrollo Infantil entre los prematuros moderados y tardíos, con y sin corregir edad gestacional y los niños nacidos de término. *EGC Edad Gestacional Corregida. **EG-NC Edad Gestacional no Corregida. ***RNT Recién Nacidos de Término. 
Tabla 3. Análisis de los puntajes de la Escala de Bayley-III de desarrollo infantil por edad gestacional corregida y área del desarrollo

\begin{tabular}{|c|c|c|c|c|}
\hline Edad corregida & Áreas desarrollo & PMT & RNT & $\mathbf{P}$ \\
\hline \multirow[t]{5}{*}{8 meses } & Promedio & $94,2 \pm 7,3$ & $94,7 \pm 8,2$ & NS \\
\hline & Cognitivo & $94 \pm 7,4$ & $97,4 \pm 8,3$ & p 0,043* \\
\hline & Lenguaje & $98,7 \pm 10,5$ & $99,7 \pm 12,1$ & NS \\
\hline & Motor & $89,9 \pm 11,1$ & $87,1 \pm 12,9$ & NS \\
\hline & $\mathrm{n}$ & 47 & 43 & \\
\hline \multirow[t]{5}{*}{18 meses } & Promedio & $102,2 \pm 9,4$ & $102,6 \pm 8,0$ & NS \\
\hline & Cognitivo & $98,5 \pm 9,4$ & $103,5 \pm 10,3$ & p $0,034^{*}$ \\
\hline & Lenguaje & $103,6 \pm 14,8$ & $103,7 \pm 13,5$ & NS \\
\hline & Motor & $104,6 \pm 11,9$ & $101,3 \pm 8,0$ & NS \\
\hline & $\mathrm{n}$ & 43 & 39 & \\
\hline \multirow[t]{5}{*}{30 meses } & Promedio & $101,2 \pm 12,3$ & $105,9 \pm 10,0$ & NS \\
\hline & Cognitivo & $102 \pm 12,8$ & $109,3 \pm 12,6$ & p 0,012* \\
\hline & Lenguaje & $102 \pm 12,8$ & $105,5 \pm 11,8$ & NS \\
\hline & Motor & $98,9 \pm 13,9$ & $103,7 \pm 13,4$ & NS \\
\hline & $\mathrm{n}$ & 41 & 37 & \\
\hline
\end{tabular}

${ }^{*} \mathrm{p}<0,05 . \mathrm{PMT}=$ Prematuros moderados $\mathrm{y}$ tardíos; $\mathrm{RNT}=$ Recién nacidos de término.

10,4 vs 100,8 \pm 9,9) (figura 1). Considerando la EG corregida, los puntajes obtenidos en la áreas de lenguaje y motricidad fueron similares entre los PMT y los RNT, no obstante el desarrollo cognitivo fue inferior en los primeros (98 \pm 10,4 en PMT vs 103,1 \pm 11,4 en RNT, $\mathrm{t}=3,69, \mathrm{gl}=248, \mathrm{p}<0,01$ ) (tabla 2), la diferencia observada fue significativa, con una potencia de prueba de 0,98 y un tamaño de efecto de 0,47 . Esta diferencia se mantuvo tanto en la evaluación de los niños de 8, como en los de 18 y 30 meses (tabla 3). Al evaluar la progresión del rendimiento cognitivo en función de la edad, ésta fue significativa para ambos grupos, pero más pronunciado en los RNT que en los PMT (figura 2).

El análisis bivariado del menor desempeño cognitivo mostró una asociación significativa con la prematuridad, género masculino, partos múltiples, menor peso de nacimiento y hospitalización en el período neonatal (tabla 4). En la regresión lineal múltiple, cuya variable dependiente fue desempeño cognitivo, sólo el género y la EG mantuvieron una asociación significativa. Las mujeres obtuvieron en promedio 4,2 puntos más que los hombres, y los RNT lograron puntajes mayores en 4,7 puntos que los PMT (tabla 5).

\section{Discusión}

Durante el período en que se realizó el estudio, la prevalencia de partos prematuros de 32 y $36^{6}$ semanas de EG fue de 7,1\% en el Centro de Salud evaluado, siendo ésta superior a las estadísticas nacionales².

Al comparar el DSM de los PMT, con niños nacidos de término sin corregir EG, los prematuros presentaron un desempeño inferior. No obstante, cuando corregimos la EG, el rendimiento fue equivalente, coincidiendo con otras publicaciones $^{14,21}$. Este resultado nos parece relevante, dado que aún no hay acuerdo en la literatura científica sobre la corrección de EG, en los grandes prematuros, al evaluar el DSM.

En el análisis por áreas del desarrollo, los PMT con EG corregida presentaron un desempeño equivalente a los RNT en las áreas de lenguaje y motriz, sin embargo, el desempeño cognitivo, a pesar de encontrarse dentro del rango esperable para la edad, fue significativamente inferior. Publicaciones recientes señalan que las principales dificultades del desarrollo de los PMT se encontrarían en el área cognitiva, manifestándose como menores habilidades escolares y necesidad de apoyo educacional; además se reporta mayor prevalencia de problemas del 


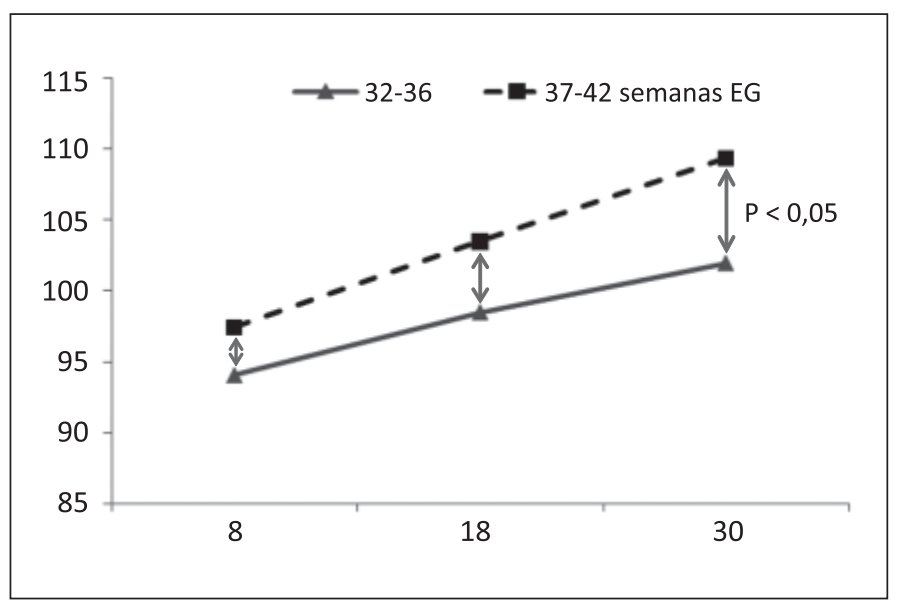

Figura 2. Progresión de los puntajes del desarrollo cogntivo de los niños nacidos prematuros moderados y tardíos y los nacidos de término (Valor de $\mathrm{p}$ en base a modelo lineal general univariado).

Tabla 4. Comparación del puntaje cognitivo según género y presencia de factores de riesgo perinatal

\begin{tabular}{|c|c|c|c|c|}
\hline & & $\begin{array}{l}\text { Número } \\
\mathrm{n}=\mathbf{2 5 0}\end{array}$ & Prom. \pm DS & $\mathbf{P}$ \\
\hline $\begin{array}{l}\text { Prematuros moderados } \\
\text { y tardíos }\end{array}$ & $\begin{array}{l}\text { Sí } \\
\text { No }\end{array}$ & $\begin{array}{l}131 \\
119\end{array}$ & $\begin{array}{c}98 \pm 10,4 \\
103,1 \pm 11,4\end{array}$ & 0,001 \\
\hline Género masculino & $\begin{array}{l}\text { Sí } \\
\text { No }\end{array}$ & $\begin{array}{l}130 \\
120\end{array}$ & $\begin{array}{l}98,2 \pm 10,60 \\
102,8 \pm 11,3\end{array}$ & 0,001 \\
\hline Gemelar & $\begin{array}{l}\text { Sí } \\
\text { No }\end{array}$ & $\begin{array}{c}67 \\
183\end{array}$ & $\begin{array}{c}96,5 \pm 9,50 \\
101,9 \pm 11,4\end{array}$ & 0,000 \\
\hline Hosp Neo* & $\begin{array}{l}\text { Sí } \\
\text { No }\end{array}$ & $\begin{array}{l}116 \\
134\end{array}$ & $\begin{array}{c}98,4 \pm 10,5 \\
102,2 \pm 11,5\end{array}$ & 0,007 \\
\hline$P E G^{* *}$ & $\begin{array}{l}\text { Sí } \\
\text { No }\end{array}$ & $\begin{array}{c}64 \\
186\end{array}$ & $\begin{array}{c}97,8 \pm 10,0 \\
101,3 \pm 11,5\end{array}$ & 0,03 \\
\hline
\end{tabular}

Prom. $=$ Promedio; DS = Desviación estándard. *Hosp Neo: Hospitalización neonatal. **PEG: pequeño para la edad gestacional.

Tabla 5. Predictores significativos de desarrollo cognitivo

\begin{tabular}{|c|c|c|c|c|c|}
\hline & \multicolumn{2}{|c|}{$\begin{array}{l}\text { Coeficientes no } \\
\text { estandarizados }\end{array}$} & \multirow{2}{*}{$\begin{array}{c}\text { Coeficientes } \\
\text { tipificados } \\
\text { Error } \\
\beta\end{array}$} & \multirow[b]{2}{*}{$\mathbf{t}$} & \multirow[b]{2}{*}{ Sig. } \\
\hline & $\begin{array}{c}\text { Coeficiente } \\
\beta\end{array}$ & $\begin{array}{l}\text { Error } \\
\text { típico }\end{array}$ & & & \\
\hline Constante & 95,07 & 4,19 & & 22,69 & 0,000 \\
\hline $\begin{array}{l}\text { Género } \\
\text { femenino }\end{array}$ & 4,20 & 1,37 & 0,19 & 3,08 & 0,002 \\
\hline RNT* & 4,74 & 1,37 & 0,21 & 3,47 & 0,001 \\
\hline
\end{tabular}

Variable dependiente: Puntaje cognitivo. *RNT: recién nacidos de término. comportamiento y Síndrome de Déficit Atencional, lo que podría contribuir a las dificultades de aprendizaje $^{10,22-24}$.

Si bien en nuestro estudio no fue de seguimiento, al comparar el rendimiento entre los niños a los 8, 18 y 30 meses corregidos, pudimos constatar una menor progresión de los puntajes cognitivos de los PMT, comparado con los RNT, con una tendencia a incrementar la brecha en función a la mayor edad de evaluación. La descripción de la evolución del DSM en los niños nacidos de término fue ampliamente descrita en una publicación previa $^{25}$. El resultado obtenido nos lleva a preguntarnos si los PMT harán su catch-up cognitivo más tardíamente, por ejemplo, en la etapa escolar como lo sugiere Chyi ${ }^{9}$, sin diferencias posteriores con los niños nacidos de término ${ }^{26}$; o bien, como plantean otros autores, las diferencias podrían continuar a lo largo de la vida, acarreando una mayor frecuencia de problemas en la esfera psiquiátrica y social en la adultez $^{27-29}$.

Para la correcta interpretación de los resultados, debemos considerar que el desarrollo es multifactorial, no pudiendo atribuir todo el riesgo de menor desempeño a la exposición prematura del cerebro al ambiente extrauterino; sino que se debe tener en consideración, entre otras variables, las noxas perinatales, a la que están expuestos los PMT con mayor frecuencia ${ }^{8}$. Estas noxas podrían desencadenar una menor organización neuronal, con consecuentes dificultades en el DSM y habilidades escolares.

Para evitar sesgos en el análisis de factores asociados al menor rendimiento, pareamos los PMT y RNT por nivel socioeconómico, edad, y género; encontramos dife- 
rencias entre las variables de riesgo perinatal relacionadas con la prematuridad, como la hospitalización en el período neonatal, peso de nacimiento, ser pequeño para la edad gestacional y gemelaridad. Como se habría esperado, de acuerdo a reportes previos, en el análisis bivariado todas estos factores se asociaron negativamente con el desempeño cognitivo, al igual que el género masculino ${ }^{11,14,15,30}$. En el modelo de regresión lineal, la EG y el género fueron las variables predictivas e independientes que mejor explicaron el resultado cognitivo. Los efectos de la gemelaridad, hospitalización y peso de nacimiento no se expresaron en este análisis por efecto de la multicolinealidad con la condición de prematuridad.

Cabe mencionar el elevado porcentaje de gemelares que aparece en nuestro grupo de PMT, lo que podría ser explicado porque el estudio fue realizado en un centro de referencia para el tratamiento de patologías de la fertilidad, lo que se asocia a embarazos múltiples y nacimientos prematuros; estudios previos han sugerido que este factor de asociaría a un menor desempeño $\operatorname{cognitivo~}^{9,23}$, coincidiendo con los resultados de nuestro análisis bivariado.

Estamos conscientes de que existen otros factores ambientales que pueden afectar el desarrollo infantil, que no fueron considerados en este estudio, lo cual podría constituir una limitante. Es probable también que los padres y/o cuidadores que aceptaron participar de la investigación hayan estado motivados, en parte, por una mayor aprehensión por la salud y desarrollo de sus niños, fenómeno que ha sido planteado por otros investigadores ${ }^{31,32}$. Sin embargo, aun considerando las limitantes descritas, nuestro estudio puede dar algunas luces sobre las diferencias en el DSM en niños nacidos PMT.

Se concluye que, durante los primeros 30 meses de vida, al corregir la EG, los PMT tienen un DSM global equivalente a los RNT, no obstante el desempeño cognitivo es inferior. Sin intervención es probable que esta diferencia se arrastre hasta la edad escolar, aunque es necesario hacer un seguimiento a largo plazo para evaluar si en nuestra población ocurre un catch-up en etapas más tardías.

El equipo de atención pediátrica debe estar alerta ante este nuevo grupo de riesgo, por lo que debería ser considerada la implementación de una norma estándar de cuidado en prematuros tardíos, diferente del que se lleva a cabo en los niños de término, con énfasis en el desarrollo cognitivo.

\section{Referencias}

1.- Shapiro-Mendoza C: Infants Born Late Preterm: Epidemiology, Trends, and Morbidity Risk. NeoReviews 2009; 10: e287-94.

2.- Departamento de Estadísticas e Información de Salud (DEIS): Ministerio de Salud, htpp://www.deis.minsal.cl

3.- American Academy of Pediatrics. Committee on Fetus and Newborn: Hospital discharge of the high-risk neonate-proposed guidelines. Pediatrics 1998; 102: 411-7.

4.- $\quad$ Raju T, Higgins $R$, Stark A, Leveno K: Optimizing Care and Outcome for Late-Preterm (Near-Term) Infants: A Summary of the Workshop Sponsored by the National Institute of Child Health and Human Development. Pediatrics September 2006; 118: 3: 1207-14.

5.- Engle W, Tomashek $K$, Wallman $C$ and the Committee on Fetus and Newborn: "Late-Preterm” Infants: A Population at Risk. Pediatrics 2007; 120: 1390-401.

6.- Shapiro-Mendoza C, Tomashek K, Kotelchuck M, et al: Effect of Late-Preterm Birth and Maternal Medical Conditions on Newborn Morbidity Risk. Pediatrics 2008; 121: e223-32.

7.- Engle W: Infants Born Late Preterm: Definition, Physiologic and Metabolic Immaturity and outcomes. NeoReviews 2009; 10 (6): e280-6.

8.- Loftin R, Habli, Snyder C, Cormier C, Lewis D, Defranco E: Late Preterm Birth. Rev Obstet Gynecol 2010; 3 (1): 10-9.

9.- Chyi LJ, Lee HC, Hintz SR, Gould JB, Sutcliffe TL: School outcome of late preterm infants: special needs and challenges for infants born at 32 to 36 weeks gestation. J Pediatr 2008; 153: 25-31.

10.- Morse SB, Zheng H, Tang Y, Roth J: Early School-Age Outcomes of Late Preterm Infants. Pediatrics 2009; 123: e622-9.

11.- Van Baar AL, Vermaas J, Knots E, De Kleine MJ, Soons $P$ : Functioning at School Age of Moderately Preterm Children Born at 32 to 36 Weeks' Gestational Age. Pediatrics 2009; 124: 251-7.

12.- Mathiasen R, Hansen B, Nybo Andersen AM, Forman $J$, Greisen G: Gestational Age and Basic School Achievements: A National Follow-up Study in Denmark. Pediatrics 2010; 126: e1553-61. 
13.- Petrini, JR, Dias, T, Mccormick MC, et al: Increased risk of adverse neurological development for late preterm infants. J Pediatr 2009; 154: 169.

14.- Romeo DM, Di Stefano A, Conversano M, et al: Neurodevelopmental outcome at 12 and 18 months in late preterm infants. Eur J Paediatr Neurol 2010; 14 (6): 503-7.

15.- Baron IS, Erickson K, Ahronovich M, Baker R, Litman $F$ : Cognitive deficit in preschoolers born late-preterm. Early Human Development 2011; 87: 115-9.

16.- Martell M, Burgueño M, Arbón G, et al: Asociación entre morbilidad neonatal y desarrollo en pretérminos a la edad escolar. Arch Pediatr Urug 2007; 78 (2): 99-109.

17.- Nomura Y, Halperin J, Newcorn J, et al: The Risk for Impaired Learning-related Abilities in Childhood and Educational Attainment Among Adults Born Near-term. Journal of Pediatric Psychology 2009; 34 (4): 406-18.

18.- Gurka M, Locasale-Crouch J, Blackman J: Long-term Cognition, Achievement, Socioemotional, and Behavioral Development of Healthy Late-Preterm Infants. Arch Pediatr Adolesc Med 2010; 164 (6): 525-32. doi:10.1001/archpediatrics.2010.83.

19.- Kalia J, Visintainer P, Brumberg H, et al: Comparison of Enrollment in Interventional Therapies Between Late-Preterm and Very Preterm Infants at 12 Months' Corrected Age. Pediatrics 2009; 123: 804-9.

20.- Bayley N: Bayley Scales of Infant and Toddler Development. Socio-Emotional Scale.Third Edition. PsychCorp. 2005.

21.- Woythaler M, Mccormick M, Smith V: Late Preterm Infants Have Worse 24-Month Neurodevelopmental Outcomes Than Term Infants. Pediatrics 2011.

22.- Talge N, Holzman C, Wang J, Lucia V, Gardiner J, Breslau N: Late-Preterm Birth and Its Association With Cognitive and Socioemotional Outcomes at 6 Years of
Age. Pediatrics 2010; 126: 6: 1124-31.

23.- Huddy CL, Johnson A, Hope PL: Educational and behavioural problems in babies of 32-35 weeks gestation. Arch Dis Child Fetal Neonatal Ed 2001; 85: F23-8.

24.- Linnet KM, Wisborg K, Agerbo E, Secher NJ, Thomsen $P H$, Henriksen TB: Gestational age, birth weight, and the risk of hyperkinetic disorder. Arch Dis Child. 2006; 91 (8): 655-60.

25.- Schonhaut L, Schonstedt M, Álvarez J, Salinas P, Armijo I: Desarrollo psicomotor en niños de nivel socioeconómico medio alto. Rev Chil Pediatr 2010; 8 (2): 122-7.

26.- Dalziel SR, Lim VK, Lambert A, et al: Psychological functioning and health-related quality of life in adulthood after preterm birth. Dev Med Child Neurol 2007; 49 (8): 597-602.

27.- Moster D, Lie Rt, Markestad T: Long-term medical and social consequences of preterm birth. N Engl J Med 2008; 359: 262.

28.- Lindström K, Winbladh B, Haglund B, Hjern A: Preterm Infants as Young Adults: A Swedish National Cohort Study. Pediatrics 2007; 120 (1): 70-7.

29.- Lindström K, Lindblad F, Hjern A: Psychiatric morbidity in adolescents and young adults born preterm: a Swedish national cohort study. Pediatrics 2009; 123 (1): e47-e53.

30.- Boulet S, Schieve L, Boyle C: Birth Weight and Health and Developmental Outcomes in US Children, 19972005. Matern Child Health J 2011; 15: 836-84.

31.- Janus $M$, Goldberg S: Factors influencing family participation in a longitudinal study: comparison of pediatric and healthy samples. J Pediatr Psychol 1997; 22: 245-62.

32.- Nøvik TS: Are postal surveys in child psychiatry feasible? Results of an epidemiological study. Nord J Psychiatry 1995; 49: 337-42. 